\title{
Analytic monoids and factorization problems
}

\author{
Jerzy Kaczorowski ${ }^{1,2}$
}

Received: 2 March 2015 / Accepted: 14 January 2016 / Published online: 3 February 2016

(C) The Author(s) 2016. This article is published with open access at Springerlink.com

\begin{abstract}
The main goal of this paper is to initiate study of analytic monoids as a general framework for quantitative theory of factorization. So far the latter subject was developed either in concrete settings, for instance in orders of number fields, or abstractly, in an axiomatic way. Some of the abstract approaches are too general to address delicate problems concerning oscillatory nature of the related counting functions, or are too restrictive in the sense that they suffer from the lack of examples except classical ones i.e. the Hilbert monoids of algebraic integers and their products. The notion of an analytic monoid is enough flexible to allow constructions of many other examples, and also ensures sufficiently rich analytic structure. In particular, we construct examples of such monoids with the associated $L$-functions being products of classical Dirichlet $L$-functions and $L$-functions of twisted irreducible unitary cuspidal automorphic representations of $G L_{d}\left(\mathbb{A}_{\mathbb{Q}}\right)$ satisfying the Ramanujan conjecture and having real coefficients. Finally, to illustrate how a typical problem from the quantitative theory of factorization can be studied in the framework of analytic monoids, we formulate several results concerning oscillations of the remainder term in the asymptotic formula for the number of irreducible elements with norms less or equal $x$, as $x$ tends to infinity.
\end{abstract}

Keywords Krull monoids $\cdot$ Selberg class $\cdot$ Factorization problems

Communicated by László Márki.

$凶 \quad$ Jerzy Kaczorowski kjerzy@amu.edu.pl

1 Faculty of Mathematics and Computer Science, Adam Mickiewicz University, 61-614 Poznań, Poland

2 Institute of Mathematics of the Polish Academy of Sciences, 00-956 Warsaw, Poland 


\section{Introduction and statement of results}

The main goal of this paper is to initiate study of analytic monoids as a general framework for quantitative theory of factorization. So far the latter subject was developed either in concrete settings, for instance in orders of number fields [18], or abstractly, in an axiomatic way [2]. We refer to [2,18] and to references cited there, for an overview of the basic theory, and to $[8,9,11,14,21]$ for a selection of more recent results. Most abstract approaches are too general to address deeper problems concerning oscillatory nature of the related counting functions. For instance arithmetic formations considered in [2] are well suited for a study of the main terms, but are not sufficiently delicate to deal with more refine problems concerning remainders. On the other hand concept of an $L$-semigroup from [21] is perfect for the latter purposes, but suffers from a lack of examples except classical ones i.e. the Hilbert monoids of algebraic integers and their products. The notion of an analytic monoid (see below for the definition) is enough flexible to allow constructions of many other examples and also ensures sufficiently rich analytic structure.

Let us set the following basic notation.

For a non-empty set $\mathcal{P}$ we denote by $\mathcal{F}(\mathcal{P})$ the free abelian monoid generated by $\mathcal{P}$. By a divisor theory of a commutative and cancellative monoid $\mathcal{S}$ with a unit element $e$ we mean a monoid homomorphism $\partial: \mathcal{S} \rightarrow \mathcal{F}(\mathcal{P})$ satisfying the following two conditions.

(D1) For every $s_{1}, s_{2} \in \mathcal{S}$ if $\partial\left(s_{1}\right)$ divides $\partial\left(s_{2}\right)$ in $\mathcal{F}(\mathcal{P})$ then $s_{1}$ divides $s_{2}$ in $\mathcal{S}$.

(D2) For every $g \in \mathcal{P}$ there exist $s_{1}, \ldots, s_{n} \in \mathcal{S}$ such that

$$
g=\operatorname{g.c.d} .\left(\partial\left(s_{1}\right), \ldots, \partial\left(s_{n}\right)\right) .
$$

The elements of $\mathcal{P}$ are called primes of $\mathcal{S}$. If $\mathrm{q}(\mathcal{F}(\mathcal{P}))$ and $\mathrm{q}(\partial(\mathcal{S}))$ denote the groups of fractions of $\mathcal{F}(\mathcal{P})$ and $\partial(\mathcal{S})$ respectively, then the quotient group

$$
C l(\mathcal{S})=\mathrm{q}(\mathcal{F}(\mathcal{P})) / \mathrm{q}(\partial(\mathcal{S}))
$$

and its order $h=\# C l(\mathcal{S})$ are called the class group and the class number of $\mathcal{S}$ respectively. In this paper we shall use the following equivalent description of the class group. For $g \in \mathcal{F}(\mathcal{P})$, let $[g]$ denote the set of all $g^{\prime} \in \mathcal{F}(\mathcal{P})$ such that $g \partial(s)=g^{\prime} \partial\left(s^{\prime}\right)$ for certain $s, s^{\prime} \in \mathcal{S}$. It is called the divisor class of $g$. Divisor classes under the multiplication $[g]\left[g^{\prime}\right]=\left[g g^{\prime}\right]$ form a group which is identified with $C l(\mathcal{S})$. We refer to the monograph [2] for the basic results on the monoids with divisor theory. Let us recall here that a monoid $\mathcal{S}$ has a divisor theory if and only if $\mathcal{S}$ is a Krull monoid (see Chap. 2 in [2]). In particular, analytic monoids as defined later on are Krull monoids.

By a norm we understand a map $\|\cdot\|: \mathcal{F}(\mathcal{P}) \rightarrow \mathbb{N}$ such that

(N1) $\|e\|=1$ and $\|a\| \neq 1$ for $a \in \mathcal{F}(\mathcal{P}) \backslash\{e\}$,

(N2) $\|a b\|=\|a\|\|b\|$ for all $a, b \in \mathcal{F}(\mathcal{P})$,

(N3) for every real positive $x$ the set $\{a \in \mathcal{F}(\mathcal{P}):\|a\| \leq x\}$ is finite and there exists a positive constant $A$ such that for every $n \geq 1$

$$
\#\{a \in \mathcal{F}(\mathcal{P}):\|a\|=n\} \ll n^{A} .
$$


It is easy to see that $\|u\|=1$ if $u \in \mathcal{S}$ is invertible. Thus if a monoid admits a norm function then necessarily $e$ is the only invertible element, i.e. it is reduced. From our point of view, restricting to such monoids yields no loss of generality since in the quantitative theory of factorization we count elements up to invertible factors. In particular, we see that for normed monoids, every divisor homomorphism $\partial$ is an injection. Therefore in such case we can assume simply that $\mathcal{S}$ is a subset of $\mathcal{F}(\mathcal{P})$, and the trivial class in $C l(\mathcal{S})$ coincides with $\mathcal{S}$. For a character $\chi$ of the class group $C l(\mathcal{S})$, we define the corresponding $L$-function as follows

$$
L(s, \mathcal{S}, \chi)=\sum_{g \in \mathcal{F}(\mathcal{P})} \frac{\chi([g])}{\|g\|^{s}} .
$$

Using (N3) it is easy to see that the series absolutely converges for $\Re(s)>A+1$. Through the paper we adopt the usual conventions used in the analytic number theory and denote the complex variable by $s=\sigma+i t$ with real $\sigma$ and $t$.

The Selberg class is the set of all Dirichlet series $F(s)=\sum_{n=1}^{\infty} a_{F}(n) n^{-s}$ which are absolutely convergent in the half-plane $\sigma>1$, have meromorphic continuation to $\mathbb{C}$ with at most one singularity at $s=1$, admit Euler product expansion and satisfy a general Riemann type functional equation. We refer to surveys $[7,10,19,20]$ for precise definition and results concerning the Selberg class. Let us remark only that this class contains, at least conjecturally, all known $L$-functions from number theory and automorphic representation theory. Basic informations on the automorphic $L$ functions which shall be used in this paper can be found in $[4,17]$.

Let $\mathcal{H}_{1 / 2}$ denote the set of all Dirichlet series

$$
H(s)=\sum_{n=1}^{\infty} \frac{h(n)}{n^{s}}
$$

with complex coefficients and such that both $H(s)$ and $1 / H(s)$ absolutely converge for $\sigma>1 / 2$ and have meromorphic continuation to $\{s \in \mathbb{C}: \sigma \geq 1 / 2\} \backslash\left\{\frac{1}{2}\right\}$ with at most a finite number of zeros and poles. In particular, possible zeros and poles of $H(s)$ with real parts $\geq 1 / 2$ must necessarily lie on the vertical line $\sigma=1 / 2$.

By an analytic monoid we mean a commutative and cancellative monoid $\mathcal{S}$ with divisor theory $\partial: \mathcal{S} \rightarrow \mathcal{F}(\mathcal{P})$ satisfying the following conditions.

(AS1) The class group $C l(\mathcal{S})$ is finite.

(AS2) There exists a norm map $\|\cdot\|: \mathcal{F}(\mathcal{P}) \rightarrow \mathbb{N}$.

(AS3) There exists a real number $\lambda$ such that for every character $\chi$ of the classgroup $C l(\mathcal{S})$ there is an $L$-function $F(s, \chi)$ from the Selberg class and $H(s, \chi) \in$ $\mathcal{H}_{1 / 2}$ satisfying

$$
L(s+\lambda, \mathcal{S}, \chi)=F(s, \chi) H(s, \chi)
$$

(AS4) The functions $F(s, \chi)$ are entire for $\chi \neq \chi_{0}$, whereas $F\left(s, \chi_{0}\right)$ has a positive degree $\left(\chi_{0}\right.$ denotes the trivial character of $\left.C l(\mathcal{S})\right)$. 
Note that $F\left(s, \chi_{0}\right)$ has a pole at $s=1$ (see the proof of Theorem 1.1 below). If this pole is simple, we call $\mathcal{S}$ a simple analytic monoid.

We call $\lambda$ and $L$-functions $F(s, \chi)$, where $\chi$ runs over the characters of the class group $C l(\mathcal{S})$, the principal shift and canonical L-functions of $\mathcal{S}$ respectively.

Theorem 1.1 The principal shift and the set of canonical L-functions of an analytic monoid $\mathcal{S}$ are uniquely determined by $\mathcal{S}$. The principal shift is non-negative.

Example 1.2 It is easy to see that $L$-semigroups in the sense of [21] are obvious examples of analytic monoids with the principal shift $\lambda=0$. In particular, Hilbert monoids of algebraic number fields are analytic monoids as well. Their canonical $L$-functions are Hecke $L$-functions, see Sect. 2.1 in [21].

Example 1.3 Let $\mathbb{P}_{4,1}$ denote the set all primes $p \equiv 1(\bmod 4)$. Moreover, let $\mathfrak{P}_{4,1}=$ $\mathbb{P}_{4,1} \times\{-1,+1\}$. Denote by $D=\mathcal{F}\left(\mathfrak{P}_{4,1}\right)$ the free abelian monoid generated by $\mathfrak{P}_{4,1}$. For every $(p, \varepsilon) \in \mathfrak{P}_{4,1}$ let $\|(p, \varepsilon)\|=p$ and $\chi((p, \varepsilon))=\varepsilon$. We extend both functions by multiplicativity to $D$, and set $\mathcal{S}_{4,1}:=\{a \in D: \chi(a)=1\}$. Obviously, the embedding $\partial: \mathcal{S}_{4,1} \rightarrow D$ is a divisor theory. The class number equals 2 . Denoting, as usual, by $\chi_{0}$ the trivial character of the class group, we have

$$
L\left(s, \mathcal{S}_{4,1}, \chi_{0}\right)=\prod_{p \equiv 1(\bmod 4)}\left(1-\frac{1}{p^{s}}\right)^{-2}=\zeta_{\mathbb{Q}(i)}(s) H(s),
$$

where $\zeta_{\mathbb{Q}(i)}(s)$ denotes the Dedekind zeta function of the quadratic number field $\mathbb{Q}(i)$, and

$$
H(s)=\left(1-\frac{1}{2^{s}}\right) \prod_{p \equiv 3(\bmod 4)}\left(1-\frac{1}{p^{2 s}}\right)^{-1} .
$$

The second character of the class group can be identified with $\chi$, and we have

$$
L\left(s, \mathcal{S}_{4,1}, \chi\right)=\prod_{p \equiv 1(\bmod 4)}\left(1-\frac{1}{p^{2 s}}\right)^{-1}
$$

We see therefore that $\mathcal{S}_{4,1}$ is an analytic monoid with principal shift 0 and canonical $L$ functions $\zeta_{\mathbb{Q}(i)}(s)$ and $\mathbb{I}(s)$ (the constant function 1 ). The corresponding $H$-functions are not a finite products of Euler factors, and thus $\mathcal{S}_{4,1}$ is not an $L$-semigroup in the sense of [21]. In a similar way one can construct infinitely many examples of analytic monoids which are not $L$-semigroups.

Example 1.4 Let $\mathcal{S}$ be a simple analytic monoid with the principal shift $\lambda=0$. Then by a standard application of the Perron formula we have for every class $X \in C l(\mathcal{S})$

$$
\#\{a \in X:\|a\| \leq x\}=\kappa x+O\left(x^{\theta}\right),
$$


with certain real $\kappa>0$ and $\theta<1$ depending on $\mathcal{S}$. Note that it is expected that $F(1) \neq 0$ for all entire $L$-functions from the Selberg class, cf. the non-vanishing conjecture in [9]. Thus all simple analytic monoids with a trivial principal shift are presumably arithmetical formations in the sense of [2], Chap. 8. On the other hand analytic monoids with positive principal shifts are not arithmetical formations. Indeed, for the former we have $\#\{a \in X:\|a\| \leq x\} \gg x^{1+\lambda}$ as $x \rightarrow \infty$ for every $X \in C l(\mathcal{S})$. This follows from the fact that $L\left(s, \mathcal{S}, \chi_{0}\right)$ has a pole of positive degree at $s=1+\lambda$.

Example 1.5 We now provide an example showing that simple analytic monoids with a trivial principal shift form a proper subclass of the class of arithmetical formations. Let us fix $(1 / 2)<\theta<1$. For every sequence $b(n), n \geq 2$, of non-negative integers such that the series $\sum_{n=2}^{\infty} b(n) n^{-\sigma}$ converges for every $\sigma>\theta$ we construct an arithmetical formation as follows. For $n \geq 2$ let $\Omega(n)$ be a finite set of cardinality $b(n)$. Moreover, let $\mathbb{P}$ denote the set of all primes. We assume that $\Omega(n) \cap \Omega\left(n^{\prime}\right)=\emptyset$ for $n \neq n^{\prime}$ and $\mathbb{P} \cap \Omega(n)=\emptyset$ for $n \geq 2$. Let

$$
\mathcal{P}=\mathbb{P} \cup \bigcup_{n=2}^{\infty} \Omega(n)
$$

and

$$
\mathcal{S}:=\mathcal{F}(\mathcal{P})
$$

For $p \in \mathcal{P}$ we put

$$
\|p\|= \begin{cases}p & \text { if } p \in \mathbb{P} \\ n & \text { if } p \in \Omega(n)\end{cases}
$$

and extend this definition to $\mathcal{S}$ by multiplicativity. The class group of $\mathcal{S}$ is trivial, and the only associated $L$-function for $\Re(s)>1$ equals

$$
\begin{aligned}
L\left(s, \chi_{0}\right) & =\prod_{p \in \mathbb{P}}\left(1-\frac{1}{p^{s}}\right)^{-1} \prod_{n=2}^{\infty}\left(1-\frac{1}{n^{s}}\right)^{-b(n)} \\
& =\zeta(s) \exp (B(s)) H(s),
\end{aligned}
$$

where $\zeta(s)$ denotes the Riemann zeta function,

$$
B(s)=\sum_{n=2}^{\infty} \frac{b(n)}{n^{s}}
$$

and

$$
H(s)=\exp \left(\sum_{n=2}^{\infty} \sum_{m=2}^{\infty} \frac{b(n)}{m n^{m s}}\right) .
$$


Obviously, $H(s)$ is holomorphic for $\Re(s)>\theta / 2$ and bounded in every closed smaller half-plane. Hence in this half-plane analytic character of $L\left(s, \chi_{0}\right)$ is totally governed by the Riemann zeta function and the behavior of $B(s)$. For generic coefficients $b(n)$ the latter function cannot be continued analytically beyond the half-plane of convergence $\Re(s)>\theta>(1 / 2)$ (see Sect. 6 below), and thus in general $\mathcal{S}$ is not an analytic monoid. On the other hand, standard application of the Perron summation formula gives

$$
\#\{a \in \mathcal{S}:\|a\| \leq x\}=\prod_{n=2}^{\infty}\left(1-\frac{1}{n}\right)^{-b(n)} x+O\left(x^{\delta}\right)
$$

as $x \rightarrow \infty$ for certain $\delta<1$. Thus $\mathcal{S}$ is an arithmetical formation in the sense of [2], Chap. 8 .

Our next result significantly extends the set of examples of analytic monoids.

For an irreducible unitary cuspidal automorphic representation $\pi$ of $G L_{d}\left(\mathbb{A}_{\mathbb{Q}}\right)$ let us denote by

$$
L(s, \pi)=\sum_{n=1}^{\infty} \frac{a_{\pi}(n)}{n^{s}}
$$

the corresponding $L$-function. If $\pi$ is written as a restricted tensor product of local representations $\pi=\pi_{\infty} \otimes \otimes_{p} \pi_{p}$, then $L(s, \pi)$ splits into the corresponding local factors

$$
L(s, \pi)=\prod_{p} L_{p}(s, \pi)
$$

where

$$
L_{p}(s, \pi)=\prod_{j=1}^{d}\left(1-\frac{\alpha_{\pi, j}(p)}{p^{s}}\right)^{-1}
$$

for certain complex numbers $\alpha_{\pi, j}(p)$. The Ramanujan conjecture asserts that $a_{\pi}(n) \ll$ $n^{\varepsilon}$ for every $\varepsilon>0$ or, equivalently, that $\left|\alpha_{\pi, j}(p)\right| \leq 1$ for all primes $p$ and all $1 \leq j \leq d$. Under the Ramanujan conjecture, $L(s, \pi)$ belongs to the Selberg class. For every primitive Dirichlet character $\chi(\bmod q)$ with $q$ coprime to the conductor of $\pi$ the twist

$$
L(s, \pi \otimes \chi)=\sum_{n=1}^{\infty} \frac{a_{\pi}(n) \chi(n)}{n^{s}}
$$

belongs to the Selberg class as well. In case when $q$ and the conductor of $\pi$ are not coprime, $L(s, \pi \otimes \chi)$ 'almost' belongs to the Selberg class in the sense that a correction of a finite number of local factors is needed. For our purposes this is completely acceptable since products of such factors belong to $\mathcal{H}_{1 / 2}$. For simplicity however we restrict ourselves to the case of coprime conductors. 
Theorem 1.6 Let $\pi$ be an irreducible unitary cuspidal automorphic representation of $G L_{d}\left(\mathbb{A}_{\mathbb{Q}}\right)$ satisfying the Ramanujan conjecture and having real coefficients $a_{\pi}(n)$. Then for every real $\lambda>1 / 2$ and every positive integer $q$ coprime to the conductor of $\pi$, there exists an analytic monoid $\mathcal{S}(\lambda)$ with the principal shift equal to $\lambda$, the class group isomorphic to $(\mathbb{Z} / q \mathbb{Z})^{\times}$and the set of canonical $L$-functions equal to

$$
L^{d}\left(s, \chi^{*}\right) L\left(s, \pi \otimes \chi^{*}\right)
$$

where $\chi$ runs over all Dirichlet characters $(\bmod q), \chi^{*}$ denotes the primitive character inducing $\chi$, and $L\left(s, \chi^{*}\right)$ denotes the classical Dirichlet L-function corresponding to $\chi^{*}$.

The proof of this theorem given in Sect. 5 below contains an explicit construction of $\mathcal{S}(\lambda)$. An analytic monoid can be called automorphic if all its canonical $L$-functions are products of automorphic $L$-functions corresponding to cusp forms. Monoids $\mathcal{S}(\lambda)$ in Theorem 1.6 can serve as examples. Let us remark that according to the main structural conjecture in the Selberg class theory, we expect that all analytic monoids are automorphic. Unfortunately, we are still very far away from being able to prove that in full generality.

\section{A sample result on factorization in analytic monoids}

In this section we shall illustrate how a typical problem from the quantitative theory of factorization can be studied in the framework of analytic monoids.

Let $\mathcal{S}$ be an analytic monoid with the principal shift $\lambda$. Let $M_{\mathcal{S}}$ denote the set of irreducible elements of $\mathcal{S}$, and

$$
M_{\mathcal{S}}(x)=\#\left\{b \in M_{\mathcal{S}}:\|b\| \leq x\right\}
$$

be the corresponding counting function. For complex $s=\sigma+i t, \sigma>1+\lambda$, let

$$
Z_{\mathcal{S}}(s)=\sum_{b \in M_{\mathcal{S}}} \frac{1}{\|b\|^{s}}
$$

be the associated zeta function. Let us write $C l(\mathcal{S})=\left\{X_{1}=\mathcal{S}, X_{2}, \ldots, X_{h}\right\}$, and for $1 \leq i \leq h, \sigma>1+\lambda$

$$
P_{i}(s)=\sum_{p \in X_{i} \cap \mathcal{P}} \frac{1}{\|p\|^{s}}
$$

Moreover, let $V$ denote the set of all sequences $\left[d_{1}, \ldots, d_{h}\right], d_{i} \in \mathbb{N} \cup\{0\}$, such that the following identity $X_{1}^{d_{1}} \ldots X_{h}^{d_{h}}=\mathcal{S}$ holds and is minimal in the sense that the product $X^{e_{1}} \ldots X_{h}^{e_{h}}$ with $0 \leq e_{i} \leq d_{i}$ equals $\mathcal{S}$ if and only if either all $e_{i}^{\prime} s$ are zero or 
$e_{i}=d_{i}$ holds for $i=1, \ldots, h$. The constant

$$
D(C l(\mathcal{S}))=\max _{\left[d_{1}, \ldots, d_{h}\right] \in V} \sum_{i=1}^{h} d_{i}
$$

is called the Davenport constant of the class group $C l(\mathcal{S})$ (compare [2,18], Chap. 5). With this notation we have

$$
Z_{\mathcal{S}}(s)=\sum_{\left[d_{1}, \ldots, d_{h}\right] \in V} \prod_{i=1}^{h} \sum_{k=0}^{d_{i}} \frac{1}{k !} \sum_{\substack{m_{1} \geq 1 \\ m_{1}+\ldots+m_{k}=d_{i}}} \ldots \sum_{\substack{m_{k} \geq 1 \\ m_{1}}} \frac{P_{i}\left(m_{1} s\right) \ldots P_{i}\left(m_{k} s\right)}{m_{1} \ldots m_{k}}
$$

This identity can be established following Sect. 8 in [8], where a special case was treated. For $\sigma>1+\lambda$ we have

$$
P_{i}(s)=\frac{1}{h} \sum_{\chi \in \widehat{C l(\mathcal{S})}} \overline{\chi\left(X_{i}\right)} \sum_{m=1}^{\infty} \frac{\mu(m)}{m} \log L\left(m s, \mathcal{S}, \chi^{m}\right),
$$

where $\mu(n)$ denotes the classical Möbius function. This gives analytic continuation of $Z_{\mathcal{S}}(s)$ to a region containing the closed half-plane $\sigma \geq 1 / 2+\lambda$ with slides starting either at $s=1+\lambda$ or $s=(\rho+\lambda) / m$ and going left to the boundary of the region. Here $\rho$ denotes a generic non-trivial zero of canonical $L$-functions $F(s, \chi)$, and $m$ is a positive integer. Let $\eta>0$ and $\delta>0$ be so small that $Z_{\mathcal{S}}(s)$ is holomorphic in the following sliced rectangle

$$
\left\{s=\sigma+i t: \frac{1}{2}+\lambda-\eta \leq \sigma \leq 2+\lambda,|t| \leq \delta\right\} \backslash\left(\frac{1}{2}+\lambda-\eta, 1+\lambda\right] .
$$

Moreover, let $\mathcal{C}$ be the path consisting of the following three parts. The line segment from $(1 / 2)+\lambda-\eta$ to $1+\lambda-\delta$ on the lower side of the real axis, the circumference $s=1+\lambda+\delta e^{i \theta},-\pi \leq \theta \leq \pi$ and the line segment from $1+\lambda-\delta$ to $(1 / 2)+\lambda-\eta$ on the upper side of the real axis. Then put

$$
T_{\mathcal{S}}(x)=\frac{1}{2 \pi i} \int_{\mathcal{C}} Z_{\mathcal{S}}(s) \frac{x^{s}}{s} d s
$$

Theorem 2.1 Let $\mathcal{S}$ be an analytic monoid with the principal shift $\lambda$. Moreover, suppose that its canonical L-functions do not vanish for $s=\sigma+i$ with

$$
\sigma>1-\frac{c_{0}}{\log \tau} \quad(\tau=|t|+10)
$$

and certain $c_{0}=c_{0}(\mathcal{S})>0$.

Then, as $x \rightarrow \infty$, we have 
(1)

$$
M_{\mathcal{S}}(x) \sim T_{\mathcal{S}}(x)
$$

(2)

$$
T_{\mathcal{S}}(x) \sim \frac{x^{1+\lambda}}{\log x} \sum_{j=0}^{\infty} \frac{P_{j}(\log \log x)}{(\log x)^{j}}
$$

where $P_{j}$ are complex polynomials of degrees $\leq D(C l(\mathcal{S})$ ) (the Davenport constant of $C l(\mathcal{S})$ ) and deg $P_{0}=C l(D(\mathcal{S}))-1$.

(3) Under the Generalized Riemann Hypothesis for canonical L-functions of $\mathcal{S}$ we have

$$
E_{\mathcal{S}}(x)=M_{\mathcal{S}}(x)-T_{\mathcal{S}}(x) \ll x^{\frac{1}{2}+\lambda+\varepsilon}
$$

for every $\varepsilon>0$.

(4) There exists a positive constant $M=M(\mathcal{S})$ such that for the error term $E_{\mathcal{S}}(x)$ we have the following omega estimate

$$
E_{\mathcal{S}}(x)=\Omega_{ \pm}\left(\frac{x^{\frac{1}{2}+\lambda}}{(\log x)^{M}}\right) .
$$

(5) For sufficiently large $T$ the error term $E_{\mathcal{S}}(x)$ changes sign $\gg \log T$ times in the interval $(1, T]$.

Let us remark that after suitable modifications, a part of assertions of Theorem 2.1 hold true for arithmetical formations. In particular, asymptotic formulae similar to (1), (2) and (3) (with $\lambda=0$ ) are proved in [2], Sect. 8.5. So, in principle, there is no novelty in this part of Theorem 2.1 (not counting the appearance of arbitrary shifts $\lambda \geq 0$ ).

With respect to the main terms of asymptotic formulae, quantitative theories of factorization in arithmetic formations and analytic monoids run parallel. Nevertheless, there are differences, even on the level of their formulations. For instance, definition of the main term in the form of a contour integral (2.4) should be modified in the case of formations. The point is that $L$-functions of formations exist on a half-plane of the form $\sigma>\theta$ for certain unspecified constant $\theta<1$, and there is no guarantee that they can be continued any further to the left. In fact, Example 1.5 shows that there exist formations with $\theta<1$ arbitrarily close to 1 , and $L$-functions for which the vertical line $\sigma=\theta$ is a natural boundary. In such cases the integrand in (2.4) is not holomorphic on the whole contour $\mathcal{C}$. Thus we have to shorten it by taking a part lying on the half-plane $\sigma \geq \theta_{1}$ for certain $\theta<\theta_{1}<1$. This is perfectly okay when dealing with the main term, since two such integrals with $\theta_{1}$ and $\theta_{2}$ respectively differ by a negligible error of size $O\left(x^{\min \left(\theta_{1}, \theta_{2}\right)}\right)$.

In contrary, proofs of (4) and (5) require finer properties of the involved $L$-functions, and the latter are not available for general formations, see Theorem 2.2 below. To 
illustrate the situation let us assume for simplicity that the class group is trivial. Then $M_{\mathcal{S}}(x)$ counts simply prime divisors with norms $\leq x$. Let $\mathcal{S}$ denote the formation constructed in Example 1.5. We have

$$
M_{\mathcal{S}}(x)=\pi(x)+\sum_{n \leq x} b(n) .
$$

According to the above remarks on the required modifications in the definition of the main term, we should replace $\mathcal{C}$ in (2.4) by a part of it lying in the half-plane $\sigma \geq \theta_{1}(>\theta)$. Then an easy computation shows that

$$
T_{\mathcal{S}}(x)=\operatorname{li}(x)-\operatorname{li}\left(x^{\theta_{1}}\right) .
$$

Suppose that the Riemann hypothesis for $\zeta(s)$ is true. Then the difference $E_{\mathcal{S}}(x)=$ $M_{\mathcal{S}}(x)-T_{\mathcal{S}}(x)$ equals

$$
\operatorname{li}\left(x^{\theta_{1}}\right)+O\left(x^{\theta+\varepsilon}\right) \quad(\varepsilon>0)
$$

which for sure is not what one would like to call a proper remainder term.

One could argue that a better choice for a main term here would be the integral $\operatorname{logarithm} \operatorname{li}(x)$ alone. Then, however, there error term would read as

$$
E_{\mathcal{S}}(x)=\sum_{n \leq x} b(n)+O(\sqrt{x} \log x),
$$

and could be dominated by partial sums of the coefficients $b(n)$. The latter can be almost arbitrary, the only restriction being that $\sum_{n \leq x} b(n) \ll x^{\theta+\varepsilon}$ for every $\varepsilon>0$ as $x \rightarrow \infty$. In particular, with a proper choice of $b(n)$ 's one could make $E_{\mathcal{S}}(x)$ of a constant sign, so that neither (4) nor (5) would hold true.

A proof of Theorem 2.1 could be conducted modifying ideas from $[6,8,9,14]$. In order to keep the size of this paper in a reasonable limits, we prefer to postpone details to a future paper, where oscillations of various remainder terms arising in the quantitative theory of factorization in analytic monoids shall be treated in a more systematic way. Here we shall outline the main idea leading to the following weakened version of the omega estimate from (4)

$$
E_{\mathcal{S}}(x)=\Omega_{ \pm}\left(x^{\frac{1}{2}+\lambda-\varepsilon}\right)
$$

as $x \rightarrow \infty$ for every $\varepsilon>0$. The main tool in the proof is the following theorem on the independence of singularities of $L$-functions from the Selberg class. Let $\mathcal{A}$ denote the set of all functions $f(s)$ holomorphic for $\sigma>1$ and having analytic continuation to $\sigma \geq(1 / 2)$ and $|t|>T_{1}$ for certain $T_{1} \geq 0$.

Theorem 2.2 For everynon-constant polynomial $P\left(X_{1}, \ldots, X_{N}\right)$ of $N$ variables with coefficients in $\mathcal{A}$, and for arbitrary L-functions $F_{1}(s), \ldots, F_{N}(s)$ from the Selberg 
class for which the logarithms $\log F_{1}, \ldots, \log F_{N}$ are linearly independent over $\mathbb{Q}$, the function

$$
p(s)=P\left(\log F_{1}(s), \ldots, \log F_{N}(s)\right)
$$

has infinitely many singularities in the half-plane $\sigma \geq \frac{1}{2}$.

This is a special case of Theorem 1 in [11].

Let $F\left(s, \chi_{1}\right), \ldots, F\left(s, \chi_{N}\right)$ be the canonical $L$-functions of $\mathcal{S}$. Without loss of generality, we can assume that their $\operatorname{logarithms} \log F\left(s, \chi_{1}\right), \ldots, \log F\left(s, \chi_{N}\right)$ are linearly independent over $\mathbb{Q}$. Otherwise, we just replace the whole set

$$
\left\{\log F\left(s, \chi_{1}\right), \ldots, \log F\left(s, \chi_{N}\right)\right\}
$$

by its maximal linearly independent subsubset. From (2.1), (2.2) and axiom (AS3) it is obvious that $Z_{\mathcal{S}}(s+\lambda)$ is of the form (2.6), with $F_{j}(s)=F\left(s, \chi_{j}\right), j=1, \ldots, N$. Thus $Z_{\mathcal{S}}(s)$ has infinitely many singularities in the half-plane $\sigma \geq \frac{1}{2}+\lambda$. Knowing this, the rest of the proof is just a standard application of the classical Landau's theorem (see, e.g. Chap. 5 of Ingham [3]) and runs as follows. Suppose that

$$
E_{\mathcal{S}}(x) \geq-A x^{\frac{1}{2}+\lambda-\varepsilon}
$$

for certain positive A and all $x \geq 1$. Without lost of generality we can assume that $\varepsilon<\eta$, where $\eta$ has the same meaning as in (2.3). Let us consider the following function defined on the positive real axis

$$
f(x)= \begin{cases}0 & \text { if } 0<x<1, \\ E_{\mathcal{S}}(x)+A x^{\frac{1}{2}+\lambda-\varepsilon} & \text { if } x \geq 1 .\end{cases}
$$

For $\sigma>1+\lambda+\delta$, where $\delta$ has the same meaning as in (2.3), its Mellin transform equals

$$
\begin{aligned}
F(s) & =\int_{0}^{\infty} f(x) x^{-s-1} d x \\
& =\int_{1}^{\infty} M_{\mathcal{S}}(x) x^{-s-1} d x-\int_{1}^{\infty} T_{\mathcal{S}}(x) x^{-s-1} d x+A \int_{1}^{\infty} x^{\frac{1}{2}+\lambda-\varepsilon-s-1} d s \\
& =\frac{1}{s} Z_{\mathcal{S}}(s)-\frac{1}{2 \pi i} \int_{\mathcal{C}} \frac{Z_{\mathcal{S}}(w)}{w(s-w)} d w+\frac{A}{s-\left(\frac{1}{2}+\lambda-\varepsilon\right)}
\end{aligned}
$$

This gives meromorphic continuation of $F(s)$ to the rectangle

$$
\left\{s: \frac{1}{2}+\lambda-\eta<\Re(s) \leq 1+\lambda+\delta,|\Im(s)|<\delta\right\}
$$


with the only singularity at $s=\frac{1}{2}+\lambda-\varepsilon$. By Landau's theorem the integral

$$
\int_{0}^{\infty} f(\xi) \xi^{-s-1} d \xi
$$

converges absolutely for $\sigma>\frac{1}{2}+\lambda-\varepsilon$. In particular, $F(s)$ is holomorphic in this half-plane. It follows also that $Z_{\mathcal{S}}(s)$ is holomorphic for $\sigma \geq \frac{1}{2}+\lambda, t \neq 0$ which, as we have seen before, is not true.

This shows that

$$
E_{\mathcal{S}}(x)=\Omega_{-}\left(x^{\frac{1}{2}+\lambda-\varepsilon}\right)
$$

The corresponding $\Omega_{+}$-estimate can be proved in a similar way, one has to change ' + ' to ' - ' in the definition of $f(x)$.

Let us remark finally, that a more refined methods needed in the proofs of (4) and (5) of Theorem 2.1, as well as some other similar results, require that the involved zeta functions have singularities of a special type, see [8,9, 14,21]. For instance, they should be isolated and be combinations of poles and logarithmic branch points. Observe, that Theorem 2.2 guarantees the existence of singularities of the desired type.

\section{Some open problems on analytic monoids}

We hope that the following list of open problems shall stimulate research on analytic monoids. Solutions of some of them could be not very hard, some of them however seem to lie quite deep.

(1) Develop quantitative theory of factorization in analytic monoids focusing on the involved error terms (estimates from above, mean value estimates of remainders, omega estimates, changes of sign, distribution of elements with prescribed factorization properties in short intervals, and so on).

(2) Give further examples (constructions) of analytic monoids. In particular, give examples with the principal shift $0<\lambda \leq 1 / 2$.

(3) Let $F\left(s, \chi_{0}\right)$ be the canonical $L$-function of an analytic monoid $\mathcal{S}$ corresponding to the trivial character of the class group. Let $d$ be its degree and let $m$ be the order of the pole at $s=1$. We can call these constants the degree and the order of $\mathcal{S}$, and denote them by $d_{\mathcal{S}}$ and $m_{\mathcal{S}}$ respectively. For instance, the monoid $\mathcal{S}(\lambda)$ in Theorem 1.6 is of degree $2 d$ and order $d$. Prove that for every $\mathcal{S}$ one has $m_{\mathcal{S}} \leq d_{\mathcal{S}}$. For a given positive integers $m \leq d$ give examples of analytic monoid with $d_{\mathcal{S}}=d$ and $m_{\mathcal{S}}=m$. In some instances this is not difficult. If $m \geq d / 2$ one can start with $\zeta^{2 m-d}(s) \zeta_{K}^{d-m}(s)$, where $\zeta_{K}(s)$ is the Dedekind zeta function of a quadratic number field $K$, and then proceed suitably modifying the proof of Theorem 1.6. There are also other easy cases.

(4) Which sets $\left\{F_{j}(s)\right\}_{j=1}^{N}$ of elements of the Selberg class are the sets of canonical $L$ functions? Give full characterization. A sufficient or necessary conditions would be interesting as well. Example 1.3 shows that they do not need to be of of the same degree. Do they have some special properties? 
(5) Characterize analytic monoids with the same sets of canonical $L$-functions (note that they do not have to be isomorphic).

(6) Characterize analytic monoids with the same $L$-function corresponding to the trivial character. In particular, describe all analytic monoids with $F\left(s, \chi_{0}\right)=\zeta(s)$ (the Riemann zeta function).

(7) Does a canonical $L$-function of an analytic monoid have a polynomial Euler Product? Probably every $L$-function from the Selberg class does, but for canonical $L$-functions this could be easier to prove, possibly assuming some additional conditions on $\mathcal{S}$. Note that for every character $\chi$ of the class group the associated $L$-function has two product expansions, the first due to multiplicativity of norm function and unique factorization in $\mathcal{F}(\mathcal{P})$

$$
L(s, \mathcal{S}, \chi)=\prod_{p \in \mathcal{P}}\left(1-\frac{\chi([p])}{\|p\|^{s}}\right)^{-1}
$$

and the second,

$$
L(s, \mathcal{S}, \chi)=\prod_{p} F_{p}(s, \chi) H(s, \chi),
$$

due to the fact that $F(s, \chi)$ belongs to the Selberg class. There is an obvious question about the exact meaning of this fact. Does this imply that the norms of prime divisors $p \in \mathcal{P}$ are powers of rational primes (probably "No"), or at least are somehow close to them (probably "Yes")?

(8) Can we relax condition (AS3) in the definition of analytic monoid assuming that the canonical $L$-functions $F(s, \chi)$ belong to the extended Selberg rather than to the Selberg class itself (possibly at the cost of sharpening (N3) by a change of the exponent $A$ to $\lambda+\varepsilon)$ ?

(9) Prove zero-free regions for canonical $L$-functions. In some cases it is possible to follow the classical method of de la Vallée Pouissin (for instance for monoids of order 1).

\section{Proof of Theorem 1.1}

Let $\sigma_{a}(\mathcal{S})$ denote the abscissa of absolute convergence of $L\left(s, \mathcal{S}, \chi_{0}\right)$, where $\chi_{0}$ is the trivial character of $C l(\mathcal{S})$. We show that $\lambda=\sigma_{a}(\mathcal{S})-1$. This will prove that $\lambda$ is uniquely determined.

We have

$$
L\left(s+\lambda, \mathcal{S}, \chi_{0}\right)=F\left(s, \chi_{0}\right) H\left(s, \chi_{0}\right),
$$

with $F\left(s, \chi_{0}\right)$ from the Selberg class and $H\left(s, \chi_{0}\right) \in \mathcal{H}_{1 / 2}$. The RHS is holomorphic for $\Re(s)>1 / 2$ except possibly for $s=1$ where could be a pole of a finite order. Suppose that there is no such a pole. Since $L\left(s+\lambda, \mathcal{S}, \chi_{0}\right)$ has non-negative coefficients, it converges absolutely for $\Re(s)>1 / 2$ by the classical Landau theorem (see for instance Theorem 11.13 in [1]). Thus 


$$
F\left(s, \chi_{0}\right)=L\left(s+\lambda, \mathcal{S}, \chi_{0}\right) / H\left(s, \chi_{0}\right)
$$

converges absolutely for $\Re(s)>1 / 2$ as well. But this is impossible since the abscissa of absolute convergence of a function from the Selberg class of a positive degree $d$ is greater or equal to $1 / 2+1 /(2 d)>1 / 2$ as trivially follows from Theorem 1 in [13]. Thus $F\left(s, \chi_{0}\right)$ has a pole at $s=1$ and hence $L\left(s, \mathcal{S}, \chi_{0}\right)$ has a pole at $1+\lambda$. This shows that $\sigma_{a}(\mathcal{S})=1+\lambda$, as claimed.

We prove now that the set of canonical $L$-functions of an analytic monoid is uniquely determined. Suppose in contrary that

$$
L(s+\lambda, \mathcal{S}, \chi)=F_{j}(s, \chi) H_{j}(s, \chi)
$$

for certain $F_{1}(s, \chi) \neq F_{2}(s, \chi)$ from the Selberg class, $H_{1}(s, \chi), H_{2}(s, \chi) \in \mathcal{H}_{1 / 2}$ and a character $\chi$ of the class group $C l(\mathcal{S})$. Hence

$$
F_{1}(s, \chi)=F_{2}(s, \chi) H(s, \chi)
$$

for $H(s, \chi) \in \mathcal{H}_{1 / 2}$. Thus zeros of $F_{1}$ and $F_{2}$ in the half-plane $\Re(s) \geq 1 / 2$ are the same up to a finite number of exceptions. The same holds for $\overline{F_{1}(\bar{s}, \chi)}$ and $\overline{F_{2}(\bar{s}, \chi)}$. Hence, by the functional equation of $L$-functions from the Selberg class we see that $F_{1}(s, \chi)$ and $F_{2}(s, \chi)$ have the same non-trivial zeros $\rho=\beta+i \gamma$ with $\gamma>0$ up to a finite number of exceptions. Now we could proceed along the lines of the proof of the main theorem in [12], i.e. using Stirling's formula and Bohr's theorem on almost periodic functions. Instead, we give an alternative proof based on $k$-functions associated to $F_{j}$ 's defined for $z$ in the upper half-plane as follows

$$
k\left(z, F_{j}(s, \chi)\right)=\sum_{\gamma>0} e^{\rho z} \quad(j=1,2),
$$

where the summation is over non-trivial zeros of $F_{j}(s, \chi)$ with positive imaginary parts. Obviously, in our case they differ by an entire function

$$
k\left(z, F_{1}(s, \chi)\right)=k\left(z, F_{2}(s, \chi)\right)+E(z, \chi)
$$

say.

Lemma 4.1 Let $F$ be an L-function from the Selberg class, and let $-\Lambda_{F}(n)$ denote Dirichlet coefficients of its logarithmic derivative, i.e.

$$
-\frac{F^{\prime}}{F}(s)=\sum_{n=1}^{\infty} \frac{\Lambda_{F}(n)}{n^{s}} \quad(\Re(s)>1) .
$$

Then the corresponding $k(z, F)$-function is holomorphic on the upper half-plane and has meromorphic continuation to the whole complex plane with the slit along the 
negative imaginary axis. The only singularities on $\mathbb{C} \backslash(-i \infty, 0]$ are simple poles at points $\pm \log n$, where $n>2$ are integers such that $\Lambda_{F}(n) \neq 0$. We have

$$
\operatorname{Res}_{z=\log n} k(z, F)=\frac{1}{2 \pi i} \Lambda_{F}(n) .
$$

Proof The lemma easily follows from Theorem 1 of [15].

Computing residues of both sides of (4.2) at $s=\log n, n \in \mathbb{N}$, with the help of Lemma 4.1 we infer that the Dirichlet coefficients of $F_{1}^{\prime} / F_{1}(s, \chi)$ and $F_{2}^{\prime} / F_{2}(s, \chi)$ are the same. Thus $F_{1}^{\prime} / F_{1}(s, \chi)=F_{2}^{\prime} / F_{2}(s, \chi)$ and hence $F_{1}(s, \chi)=c F_{2}(s, \chi)$ for certain complex number $c$. Comparing the first Dirichlet coefficient of both sides we see that $c=1$, and hence $F_{1}(s, \chi)=F_{2}(s, \chi)$, a contradiction.

To prove the last assertion suppose in contrary that the principal shift $\lambda$ is negative. Take the trivial character $\chi_{0}$ of the class group and compare coefficients of Dirichlet series of both sides of (4.1). With obvious notation we have

$$
a_{L}(n) n^{|\lambda|} \leq \sum_{d \mid n}\left|a_{F}(n) a_{H}\left(\frac{n}{d}\right)\right| \ll n^{\varepsilon}
$$

for every positive $\varepsilon$ since both $F$ and $H$ satisfy Ramanujan condition. But $a_{L}(n)$ is the number of $g \in \mathcal{F}$ with $\|g\|=n$, and hence is a non-negative integer. Thus (4.3) implies $n^{|\lambda|} \ll n^{\varepsilon}$ if $a_{L}(n) \neq 0$. This is impossible if $\varepsilon<|\lambda|$ and $n$ sufficiently large. Thus $L\left(s, \mathcal{S}, \chi_{0}\right)$ is a Dirichlet polynomial. Then, as at the beginning of the proof, we conclude that $F\left(s, \chi_{0}\right)$ converges absolutely for $\Re(s)>1 / 2$, which is impossible. Theorem 1.1 follows.

\section{Proof of Theorem 1.6}

Let $a_{\pi}(n)$ and $\alpha_{\pi, j}(p)$ have the same meaning as in (1.1) and (1.2) respectively. Let us put

$$
\alpha_{\pi, j}^{*}(p)= \begin{cases}\alpha_{\pi, j}(p) & \text { if } 1 \leq j \leq d \\ 1 & \text { if } d+1 \leq j \leq 2 d\end{cases}
$$

and

$$
L^{*}(s, \pi)=\prod_{p} \prod_{j=1}^{2 d}\left(1-\frac{\alpha_{\pi, j}^{*}(p)}{p^{s}}\right)^{-1}
$$

Obviously,

$$
L^{*}(s, \pi)=\zeta^{d}(s) L(s, \pi)
$$


where $\zeta(s)$ is the familiar Riemann zeta function. For $\Re(s)>1$ we write

$$
L^{*}(s, \pi)=\sum_{n=1}^{\infty} \frac{a_{\pi}^{*}(n)}{n^{s}}
$$

Lemma 5.1 For all primes $p$ we have

$$
a_{\pi}^{*}(p) \geq 0
$$

and

$$
\frac{1}{2} a_{\pi}^{*}(p)\left(a_{\pi}^{*}(p)+1\right) \leq a_{\pi}^{*}\left(p^{2}\right)+d a_{\pi}^{*}(p)
$$

Proof Comparing coefficients of both sides of the identity

$$
\sum_{n=1}^{\infty} \frac{a_{\pi}^{*}(n)}{n^{s}}=\prod_{p} \prod_{j=1}^{2 d}\left(1-\frac{\alpha_{\pi, j}^{*}(p)}{p^{s}}\right)^{-1}
$$

we obtain

$$
a_{\pi}^{*}(p)=\sum_{j=1}^{2 d} \alpha_{\pi, j}^{*}(p)=d+\sum_{j=1}^{d} \alpha_{\pi, j}(p)
$$

and

$$
a_{\pi}^{*}\left(p^{2}\right)=\sum_{j=1}^{d} \alpha_{\pi, j}(p)\left(\alpha_{\pi, j}(p)+d\right)+\sum_{j_{1}=2}^{d} \sum_{j_{2}=1}^{j_{1}-1} \alpha_{\pi, j_{1}}(p) \alpha_{\pi, j_{2}}(p)+\frac{1}{2} d(d+1)
$$

Thus

$$
a_{\pi}^{*}(p) \geq d-\sum_{j=1}^{d}\left|\alpha_{\pi, j}(p)\right| \geq 0
$$

and (5.1) follows. Moreover, after some easy computations we obtain

$$
\begin{aligned}
& a_{\pi}^{*}\left(p^{2}\right)+d a_{\pi}^{*}(p)-\frac{1}{2} a_{\pi}^{*}(p)\left(a_{\pi}^{*}(p)+1\right) \\
& \quad=d^{2}+\frac{1}{2} \sum_{j=1}^{d} \alpha_{\pi, j}(p)\left(\alpha_{\pi, j}(p)+2 d-1\right) \geq d^{2}-d^{2}=0,
\end{aligned}
$$

and (5.2) follows. 
Let $\lambda>0$ be fixed. For primes $p$ let us put

$$
A_{\lambda}(p)=\left[a_{\pi}^{*}(p) p^{\lambda}\right]
$$

and

$$
B_{\lambda}(p)=\left[\left(a_{\pi}^{*}\left(p^{2}\right)+d a_{\pi}^{*}(p)\right) p^{2 \lambda}\right] .
$$

According to Lemma 5.1 they are non-negative integers.

Lemma 5.2 For $p \geq 3^{1 / \lambda}$ we have

$$
\frac{1}{2} A_{\lambda}(p)\left(A_{\lambda}(p)+1\right) \leq B_{\lambda}(p)
$$

Proof We can assume that

$$
a_{\pi}^{*}(p) \geq p^{-\lambda}
$$

since otherwise the assertion is trivial. If this inequality holds we have using Lemma 5.1

$$
\begin{aligned}
\frac{1}{2} A_{\lambda}(p)\left(A_{\lambda}(p)+1\right) & \leq \frac{1}{2} a_{\pi}^{*}(p) p^{\lambda}\left(a_{\pi}^{*}(p) p^{\lambda}+1\right) \\
& \leq\left(a_{\pi}^{*}\left(p^{2}\right)+d a_{\pi}^{*}(p)\right) p^{2 \lambda}-\frac{1}{2} a_{\pi}^{*}(p)\left(p^{\lambda}-1\right) p^{\lambda} \\
& \leq B_{\lambda}(p)+1-\frac{1}{2} a_{\pi}^{*}(p)\left(p^{\lambda}-1\right) p^{\lambda}
\end{aligned}
$$

Using (5.3) we have

$$
\frac{1}{2} a_{\pi}^{*}(p)\left(p^{\lambda}-1\right) p^{\lambda} \geq \frac{1}{2}\left(p^{\lambda}-1\right) \geq 1
$$

if $p \geq 3^{1 / \lambda}$, and the lemma follows.

Let

$$
C_{\lambda}(p)=B_{\lambda}(p)-\frac{1}{2} A_{\lambda}(p)\left(A_{\lambda}(p)+1\right)
$$

For $p \geq 3^{1 / \lambda}$ this is a non-negative integer.

For $\Re(s)>1+\lambda$ we put

$$
G(s)=\prod_{p}\left(1-\frac{1}{p^{s}}\right)^{-A_{\lambda}(p)}\left(1-\frac{1}{p^{2 s}}\right)^{-C_{\lambda}(p)} .
$$


Lemma 5.3 For $\lambda>1 / 2$ we have

$$
G(s+\lambda)=\zeta^{d}(s) \zeta^{d^{2}}(2 s) L(s, \pi) L^{d}(2 s, \pi) H(s),
$$

where $H(s)$ is a Dirichlet series such that both $H(s)$ and $1 / H(s)$ converge absolutely for $\Re(s)>(1 / 2)-\delta$ with certain positive $\delta$.

Proof Let $G_{p}(s)$ denote the local factor of $G(s)$ corresponding to a prime $p$. For $\sigma=\mathfrak{l}(s)>0$ we have

$$
\begin{aligned}
\log G_{p}(s+\lambda) & =-A_{\lambda}(p) \log \left(1-\frac{1}{p^{s+\lambda}}\right)-C_{\lambda}(p) \log \left(1-\frac{1}{p^{2 s+2 \lambda}}\right) \\
& =\frac{A_{\lambda}(p)}{p^{s+\lambda}}+\frac{C_{\lambda}(p)}{p^{2 s+2 \lambda}}+O\left(\frac{1}{p^{2 \sigma+\lambda}}\right) \\
& =\frac{a_{\pi}^{*}(p)}{p^{s}}+\frac{a_{\pi}^{*}\left(p^{2}\right)+d a_{\pi}^{*}(p)-\frac{1}{2} a_{\pi}^{*}(p)^{2}}{p^{2 s}}+O\left(\frac{1}{p^{\sigma+\lambda}}\right) .
\end{aligned}
$$

We have also

$$
\begin{aligned}
\log L_{p}^{*}(s, \pi) & =\log \left(1+\frac{a_{\pi}^{*}(p)}{p^{s}}+\frac{a_{\pi}^{*}\left(p^{2}\right)}{p^{2 s}}+O\left(\frac{1}{p^{3 \sigma}}\right)\right) \\
& =\frac{a_{\pi}^{*}(p)}{p^{s}}+\frac{a_{\pi}^{*}\left(p^{2}\right)-\frac{1}{2} a_{\pi}^{*}(p)^{2}}{p^{2 s}}+O\left(\frac{1}{p^{3 \sigma}}\right) .
\end{aligned}
$$

Thus

$$
\log G_{p}(s+\lambda)=\log \left(L_{p}^{*}(s, \pi) L_{p}^{*}(2 s, \pi)^{d}\right)+O\left(\frac{1}{p^{3 \sigma}}+\frac{1}{p^{\sigma+\lambda}}\right) .
$$

Note that the $O$-term in the above formula is in fact a Dirichlet series supported on positive powers of $p$. Taking exponentials of both sides and then product over all primes we arrive at $(5.5)$ with $\delta=\min (1 / 6, \lambda-1 / 2)$.

From this point on we assume that $\lambda>1 / 2$. For primes $p>\max (11, q)\left(>3^{1 / \lambda}\right)$ let $\Omega(p)$ and $\Omega\left(p^{2}\right)$ be finite sets such that

$$
\# \Omega(p)=A_{\lambda}(p), \quad \# \Omega\left(p^{2}\right)=C_{\lambda}(p)
$$

and for all primes $p_{1}, p_{2}$ and $l_{1}, l_{2} \in\{1,2\}$

$$
\Omega\left(p_{1}^{l_{1}}\right) \cap \Omega\left(p_{2}^{l_{2}}\right)=\varnothing \quad \text { if } \quad p_{1}^{l_{1}} \neq p_{2}^{l_{2}} .
$$

Moreover, let

$$
\mathcal{P}=\bigcup_{p>\max (11, q)}\left(\Omega(p) \cup \Omega\left(p^{2}\right)\right)
$$


and let $\mathcal{F}=\mathcal{F}(\mathcal{P})$ be the free abelian monoid generated by $\mathcal{P}$. We define the norm function on $\mathcal{P}$ by putting

$$
\|g\|= \begin{cases}p & \text { if } g \in \Omega(p) \\ p^{2} & \text { if } g \in \Omega\left(p^{2}\right)\end{cases}
$$

and then we extend it multiplicatively to the whole monoid $\mathcal{F}(\mathcal{P})$.

We define the monoid $\mathcal{S}(\lambda)$ as follows

$$
\mathcal{S}(\lambda)=\{g \in \mathcal{F}(\mathcal{P}):\|g\| \equiv 1(\bmod q)\} .
$$

We are going to show now that the natural embedding

$$
\partial: \mathcal{S}(\lambda) \rightarrow \mathcal{F}(\mathcal{P})
$$

is a divisor theory.

Lemma 5.4 For every $0<c_{0}<d$ and every $(a, q)=1$ there exist infinitely many primes $p \equiv a(\bmod q)$ such that $a_{\pi}^{*}(p) \geq c_{0}$. In particular, there exist infinitely many primes $p \equiv a(\bmod q)$ such that $\Omega(p) \neq \emptyset$.

Proof For $\sigma>1$ we have

$$
\begin{aligned}
\sum_{p \equiv a(\bmod q)} \frac{a_{\pi}^{*}(p)}{p^{\sigma}}= & \frac{1}{\varphi(q)} \log L^{*}(\sigma, \pi) \\
& +\frac{1}{\varphi(q)} \sum_{\chi \neq \chi_{0}} \overline{\chi(a)} \log L^{*}\left(\sigma, \pi \otimes \chi^{*}\right)+O(1) .
\end{aligned}
$$

From the main theorem of [5] it easily follows that $L^{*}\left(\sigma, \pi \otimes \chi^{*}\right) \neq 0$ if $\chi \neq \chi_{0}$ and $\sigma \geq 1$. For $\chi=\chi_{0}$ there is a pole at $s=1$. The order is $d$ if $\pi$ is non-trivial or 2 if $L(s, \pi)$ reduces to the Riemann zeta function. Thus for $\sigma>1$ we have

$$
\sum_{p \equiv a(\bmod q)} \frac{a_{\pi}^{*}(p)}{p^{\sigma}} \geq \frac{d}{\varphi(q)} \log \frac{1}{\sigma-1}+O(1),
$$

and the lemma easily follows since

$$
\sum_{p \equiv a(\bmod q)} \frac{1}{p^{\sigma}} \sim \frac{1}{\varphi(q)} \log \frac{1}{\sigma-1}
$$

as $\sigma \rightarrow 1^{+}$.

In order to check that the embedding $\partial: \mathcal{S}(\lambda) \rightarrow \mathcal{F}(\mathcal{P})$ is a divisor theory we show that 
(1) for every $g_{1}, g_{2} \in \mathcal{S}(\lambda)$ if $g_{1}$ divides $g_{2}$ in $\mathcal{F}(\mathcal{P})$ then $g_{1}$ divides $g_{2}$ in $\mathcal{S}(\lambda)$.

(2) For every $g \in \mathcal{P}$ there exist $g_{1}, \ldots, g_{n} \in \mathcal{S}(\lambda)$ such that $g$ is the greatest common divisor of $g_{1}, \ldots, g_{n}$.

Proof of (1) is easy. If $g_{2}=g \cdot g_{1}$ for $g_{1}, g_{2} \in \mathcal{S}(\lambda)$ and $g \in \mathcal{F}(\mathcal{P})$ then $\|g\| \equiv$ $\|g\| \cdot\left\|g_{1}\right\|(\bmod q) \equiv\left\|g \cdot g_{1}\right\|(\bmod q) \equiv\left\|g_{2}\right\|(\bmod q) \equiv 1(\bmod q)$. Thus $g \in \mathcal{S}(\lambda)$, and (1) follows.

To show (2) fix arbitrary $g \in \mathcal{P}$. We have $\|g\|=p^{l}, l \in\{1,2\}, p>q$. Hence $\|g\| \equiv a(\bmod q)$ for certain $(a, q)=1$. Let $\bar{a}$ denote the multiplicative inverse of $a(\bmod q)$. By Lemma 5.4 there exist infinitely many primes $\ell \equiv \bar{a}(\bmod q)$ with $\Omega(\ell) \neq \varnothing$. Take two such primes $\ell_{1} \neq \ell_{2}$ and two elements $g_{j}=g \cdot g_{j}^{\prime}, g_{j}^{\prime} \in \Omega\left(\ell_{j}\right)$, $j=1,2$. Then $g_{1}, g_{2} \in \mathcal{S}(\lambda), g=$ g.c.d. $\left(g_{1}, g_{2}\right)$, and (2) follows even in a stronger form $(n=2)$.

Suppose that $g_{1}, g_{2} \in \mathcal{F}(\mathcal{P})$ belong to the same divisor class in $\operatorname{Cl}(\mathcal{S}(\lambda))$. Then $\left\|g_{1}\right\| \equiv\left\|g_{2}\right\|(\bmod q)$. Hence we have a group homomorphism

$$
\phi: C l(\mathcal{S}(\lambda)) \ni X=[g] \mapsto\|g\|(\bmod q) \in(\mathbb{Z} / q \mathbb{Z})^{\times}
$$

From the definition of $\mathcal{S}(\lambda)$ we see that the kernel of this homomorphism is trivial, whereas Lemma 5.4 easily implies that it is onto. Thus the class group of $\mathcal{S}(\lambda)$ is isomorphic to $(\mathbb{Z} / q \mathbb{Z})^{\times}$. In particular, characters of the class group can be identified with Dirichlet characters $(\bmod q)$. For simplicity we shall use the same symbol $\chi$ to denote class group character and the corresponding Dirichlet character $\chi(\bmod q)$. For $\Re(s)>1+\lambda$ we have

$$
L(s, \mathcal{S}, \chi)=\sum_{g \in \mathcal{F}} \frac{\chi([g])}{\|g\|^{s}}=\sum_{n=1}^{\infty} \frac{a_{\mathcal{S}}(n) \chi(n)}{n^{s}},
$$

where $a_{\mathcal{S}}(n)$ denotes the number of $g \in \mathcal{F}(\mathcal{P})$ with $\|g\|=n$. The last expression is equal to

$$
\prod_{p>\max (11, q)}\left(1-\frac{\chi(p)}{p^{s}}\right)^{-A_{\lambda}(p)}\left(1-\frac{\chi(p)^{2}}{p^{2 s}}\right)^{-C_{\lambda}(p)}=G\left(s, \chi^{*}\right) H(s, \chi),
$$

where $G\left(s, \chi^{*}\right)$ is the twist of $G(s)$ defined in (5.4) by $\chi^{*}$ and $H(s, \chi) \in \mathcal{H}_{1 / 2}$ is a finite product of inverses of local factors corresponding to primes less than or equal to $\max (11, q)$ and not dividing the conductor of $\chi$. Since $\zeta^{d^{2}}(2 s) L^{d}(2 s, \pi)$ and all its twists $L^{d^{2}}\left(2 s, \chi^{*}\right) L^{d}\left(2 s, \pi \otimes \chi^{*}\right)$ belong to $\mathcal{H}_{1 / 2}$, we conclude using Lemma 5.3 that

$$
L(s+\lambda, \mathcal{S}, \chi)=L^{d}\left(s, \chi^{*}\right) L^{d}\left(s, \pi \otimes \chi^{*}\right) H_{1}(s, \chi)
$$

for certain $H_{1}(s, \chi) \in \mathcal{H}_{1 / 2}$. Hence $L^{d}\left(s, \chi^{*}\right) L^{d}\left(s, \pi \otimes \chi^{*}\right)$, where $\chi$ runs over Dirichlet characters $(\bmod q)$ are canonical $L$-functions of $\mathcal{S}(\lambda)$, and the result follows. 


\section{An auxiliary result on Dirichlet series}

In this section we briefly discuss a result on Dirichlet series which was mentioned in Example 1.5. Its formulation and proof use some basic facts from general topology related to the Baire category theorem, and can be found for instance in Chap. 6 of Kelley's classic monograph [16]. Let us recall that every countable union of closed and nowhere dense sets in a complete metric space is called a meager and is interpreted as a 'small' set, whereas its complement, called co-meager or residual, as 'large'.

Let $0<\theta<1$ be fixed, and let $X(\theta)$ denote the set of all sequences $\mathbf{b}=(b(n))_{n=1}^{\infty}$ of non-negative integers such that

$$
\sum_{n=1}^{\infty} \frac{b(n)}{n^{\sigma}}<\infty
$$

for all $\sigma>\theta$. For two sequences $\mathbf{b}, \mathbf{c} \in X(\theta)$ we define the distance between them by the following formula

$$
\rho(\mathbf{b}, \mathbf{c})=\sum_{m=1}^{\infty} \frac{1}{2^{m}} \min \left(\sum_{n=1}^{\infty} \frac{|b(n)-c(n)|}{n^{\theta+\frac{1}{m}}}, 1\right) .
$$

It is easy to see that $(X(\theta), \rho)$ is a complete metric space. For $\mathbf{b} \in X(\theta)$ we put

$$
f(s, \mathbf{b})=\sum_{n=1}^{\infty} \frac{b(n)}{n^{s}} .
$$

Obviously, $f(s, \mathbf{b})$ is a holomorphic function for $\sigma=\Re(s)>\theta$.

Proposition 6.1 The set of $\mathbf{b} \in X(\theta)$ for which the vertical line $\sigma=\theta$ is a natural boundary of $f(s, \mathbf{b})$ is residual in $X(\theta)$, in particular it is dense.

In the proof we shall need the following lemma.

Lemma 6.2 For every $t_{0} \in \mathbb{R}$ there exists $\mathbf{b}^{t_{0}} \in X(\theta)$ such that

$$
\lim _{\sigma \rightarrow \theta^{+}}\left|f\left(\sigma+i t_{0}, \mathbf{b}^{t_{0}}\right)\right|=\infty
$$

Proof Given $t_{0}$ we construct $\mathbf{b}^{t_{0}}$ as follows. Let for $\xi \geq 1$

$$
\phi(\xi)=\xi^{\theta}\left(1+\alpha \cos \left(t_{0} \log \xi\right)\right),
$$

where

$$
\alpha=\alpha\left(t_{0}\right)=\frac{\theta}{2\left(1+\left|t_{0}\right|\right)} .
$$


Obviously, the derivative $\phi^{\prime}(\xi)$ is positive for all $\xi>1$, and $\phi(\xi) \rightarrow \infty, \phi^{\prime}(\xi) \rightarrow 0$ as $\xi \rightarrow \infty$. We deduce that there exists a sequence of positive integers $M_{m}, m \geq 1$, such that

$$
\phi\left(M_{m}\right)=m+O(1)
$$

for all $m \geq 1$. We have

$$
\sum_{M_{m} \leq \xi} 1=\phi(\xi)+O(1)
$$

We put $\mathbf{b}^{t_{0}}=(b(n))_{n=1}^{\infty}$, where

$$
b(n)= \begin{cases}1 & \text { if } n=M_{m} \text { for certain } m \geq 1 \\ 0 & \text { otherwise }\end{cases}
$$

Recalling (6.1) we have for $\sigma>\theta$

$$
\begin{aligned}
f\left(s, \mathbf{b}^{t_{0}}\right) & =\sum_{m=1}^{\infty} \frac{1}{M_{m}^{s}}=s \int_{1}^{\infty} \sum_{M_{m} \leq \xi} 1 \frac{d \xi}{\xi^{s+1}} \\
& =s \int_{1}^{\infty} \phi(\xi) \frac{d \xi}{\xi^{s+1}}+h(s),
\end{aligned}
$$

where $h(s)$ is holomorphic for $\sigma>0$. A direct computation shows that the last integral equals

$$
\frac{1}{s-\theta}+\frac{\alpha}{2} \frac{1}{s-\left(\theta+i t_{0}\right)}+\frac{\alpha}{2} \frac{1}{s-\left(\theta-i t_{0}\right)} .
$$

Thus $f\left(s, \mathbf{b}^{t_{0}}\right)$ has a pole at $s=\theta+i t_{0}$, and the lemma follows.

Now we can complete the proof of Proposition 6.1. For a fixed positive integer $k$, and a rational number $\eta \in \mathbb{Q}$, let $Q=Q(k, \eta)$ denote the square defined by the following inequalities

$$
\theta<\Re(s)<\theta+\frac{1}{k}, \quad|\Im(s)-\eta|<\frac{1}{2 k} .
$$

For every such $Q$ and every positive integer $l$ let

$$
\Omega(Q, l)=\{\mathbf{b} \in X(\theta):|f(s, \mathbf{b})| \leq l \text { for all } s \in Q\}
$$

It is easy to see that $\Omega(Q, l)$ is closed in the topology induced by the metric $\rho$.

We shall prove that is it also nowhere dense. Indeed, for every $\mathbf{b} \in \Omega(Q, l)$ and every positive $\varepsilon$ let us define $\mathbf{b}_{\varepsilon} \in X(\theta)$ as follows. First we apply Lemma 6.2 for 
$t_{0}=\eta$. We deduce that there exists $\mathbf{b}^{\eta} \in X(\theta)$ such that $f\left(s, \mathbf{b}^{\eta}\right)$ is unbounded in $Q$. Then, let us fix $M$ so large that $\sum_{m>M} 1 / 2^{m}<\varepsilon / 2$. Moreover, let $N$ be so large that

$$
\sum_{n>N} \frac{b(n)}{n^{\theta+\frac{1}{M}}}<\frac{\varepsilon}{2} \text { and } \sum_{n>N} \frac{b^{\eta}(n)}{n^{\theta+\frac{1}{M}}}<\frac{\varepsilon}{2} .
$$

For such $N$ let $\mathbf{b}_{\varepsilon}=\left(b_{\varepsilon}(n)\right)_{n=1}^{\infty}$, where

$$
b_{\varepsilon}(n)= \begin{cases}b(n) & \text { if } 1 \leq n \leq N \\ b^{\eta}(n) & \text { if } n>N\end{cases}
$$

One easily checks that $\rho\left(\mathbf{b}, \mathbf{b}_{\varepsilon}\right)<\varepsilon$. Since $\mathbf{b}_{\varepsilon} \notin \Omega(Q, l)$, we see that the interior of $\Omega(Q, l)$ is empty, i.e. $\Omega(Q, l)$ is nowhere dense.

Applying the Baire category theorem we see that the set

$$
\bigcup_{\eta \in \mathbb{Q}} \bigcup_{k \in \mathbb{N}} \bigcup_{l \in \mathbb{N}} \Omega(Q(k, \eta), l)
$$

is a meager in $X(\theta)$. Observe that for every $\mathbf{b}$ from the complement set the function $f(s, \mathbf{b})$ is unbounded in every square $Q(k, \eta), \eta \in \mathbb{Q}, k \in \mathbb{N}$, and the Proposition follows.

Acknowledgements The author thanks an anonymous referee for valuable comments on the initial version of this paper. The author was partially supported by Grant 2013/11/B/ST1/02799 from the National Science Center (Poland).

Open Access This article is distributed under the terms of the Creative Commons Attribution 4.0 International License (http://creativecommons.org/licenses/by/4.0/), which permits unrestricted use, distribution, and reproduction in any medium, provided you give appropriate credit to the original author(s) and the source, provide a link to the Creative Commons license, and indicate if changes were made.

\section{References}

1. Apostol, T.M.: Introduction to Analytic Number Theory. Springer, New York (1976)

2. Geroldinger, A., Halter-Koch, F.: Non-unique Factorizations. Algebraic, Combinatorial and Analytic Theory. Pure and Applied Mathematics, vol. 278. Chapman \& Hall, Boca Raton (2006)

3. Ingham, A.E.: The Distribution of Prime Numbers. Cambridge University Press, Cambridge (1932)

4. Iwaniec, H., Kowalski, E.: Analytic Number Theory. American Mathematical Society Colloquium Publications, vol. 53. American Mathematical Society, Providence (2004)

5. Jacquet, H., Shalika, J.A.: A non-vanishing theorem for zeta functions of $G L_{n}$. Invent. Math. 38, 1-16 (1976)

6. Kaczorowski, J.: Some remarks on factorization in algebraic number fields. Acta Arith. 43, 53-68 (1983)

7. Kaczorowski, J.: Axiomatic theory of $L$-functions: the Selberg class. In: Perelli, A., Viola, C. (eds.) Analytic Number Theory. C.I.M.E. Summer School, Cetraro (Italy) 2002, pp. 133-209. Springer, L.N. 1891 (2006)

8. Kaczorowski, J.: On the distribution of irreducible algebraic integers. Monatsh. Math. 156(1), 47-71 (2009)

9. Kaczorowski, J.: $\Omega$-estimates related to irreducible algebraic integers. Math. Nachr. 283(9), 1291-1303 (2010) 
10. Kaczorowski, J., Perelli, A.: The Selberg class: a survey. In: Györy, K., et al., (eds.) Number Theory in Progress. Proceeding of the Conference in Honor of A.Schinzel, pp. 953-992. de Gruyter, Berlin (1999)

11. Kaczorowski, J., Perelli, A.: On a functional independence of singularities of a class of Dirichlet series. Am. J. Math. 120, 289-303 (1998)

12. Kaczorowski, J., Perelli, A.: Strong multiplicity one for the Selberg class. C. R. Acad. Sci. Paris 332, 963-968 (2001)

13. Kaczorowski, J., Perelli, A.: On the structure of the Selberg class, VI: non-linear twists. Acta Arith. 116, 315-341 (2005)

14. Kaczorowski, J., Pintz, J.: Oscillatory properties of arithmetical functions, II. Acta Math. Hung. 49(34), 441-453 (1987)

15. Kaczorowski, J., Ramaré, O.: Almost periodicity of some error terms in prime number theory. Acta Arith. 106(3), 277-297 (2003)

16. Kelley, J.L.: General Topology, GTM 27. Springer, Berlin (1975)

17. Michel, P.: Analytic number theory and families of $L$-functions. Automorphic Forms and Applications. IAS/Park City Mathematics Series, vol. 12, pp. 181-295. American Mathematical Society, Providence (2007)

18. Narkiewicz, W.: Elementary and Analytic Theory of Algebraic Numbers. Springer, Berlin (2004)

19. Perelli, A.: A survey of the Selberg class of $L$-functions, I. Milan J. Math. 73, 19-52 (2005)

20. Perelli, A.: A survey of the Selberg class of $L$-functions, II. Riv. Mat. Univ. Parma 7(3), 83-118 (2004)

21. Radziejewski, M.: The asymptotic behaviour of the counting functions of $\Omega$-sets in arithmetical semigroups. Acta Arith. 163(2), 179-198 (2014) 